\title{
Circuit
}

Musiques contemporaines

\section{Compléments biographiques}

\section{Pour la suite des recherches autour de l'oeuvre de Gilles Tremblay}

\section{Marie-Thérèse Lefebvre}

Volume 20, numéro 3, 2010

Gilles Tremblay, ou le plain-chant contemporain

URI : https://id.erudit.org/iderudit/044863ar

DOI : https://doi.org/10.7202/044863ar

Aller au sommaire du numéro

Éditeur(s)

Les Presses de l'Université de Montréal

ISSN

1183-1693 (imprimé)

1488-9692 (numérique)

Découvrir la revue

Citer ce document

Lefebvre, M.-T. (2010). Compléments biographiques : pour la suite des recherches autour de l'oeuvre de Gilles Tremblay. Circuit, 20(3), 91-103. https://doi.org/10.7202/044863ar d'utilisation que vous pouvez consulter en ligne.

https://apropos.erudit.org/fr/usagers/politique-dutilisation/ 


\title{
Compléments biographiques
}

\section{Pour la suite des recherches autour de l'oeuvre de Gilles Tremblayl}

\author{
Dossier préparé par Marie-Thérèse Lefebvre
}

\section{a) Avant-propos}

La saison artistique montréalaise 2009-2010 a été riche en événements musicaux. Les nombreuses activités reliées à l'œuvre du compositeur québécois Gilles Tremblay dans une Série Hommage, initiative de la Société de musique contemporaine du Québec, ont particulièrement retenu l'attention. Son directeur artistique, Walter Boudreau a, en effet, choisi de consacrer cette dernière saison à cet immense compositeur en conviant tous les intervenants des milieux musical, artistique et intellectuel intéressés à la musique de création à insérer dans leur programmation une activité mettant en relief la production de ce créateur exceptionnel.

Le résultat a été impressionnant. Outre les œuvres présentées dans de multiples concerts ${ }^{2}$, incluant la création d'un opéra3 et d'une œuvre pour voix et orchestre ${ }^{4}$, le public a pu participer à une exposition d'œuvres visuelles inspirées de la musique de Tremblay5. Les écoliers du Québec n’ont pas été oubliés: la SMCQ leur a proposé un livre illustré, et le Centre de musique canadienne a commandé un arrangement pour jeunes harmonies scolaires ${ }^{6}$. Pour clore la saison, Sylvain Caron et Marie-Thérèse Lefebvre ont organisé, sous l'égide de la Société québécoise de recherche en musique, une journée Tremblay ${ }^{7}$ dont les actes seront éventuellement publiés dans ses Cahiers.

Que reste-t-il alors à découvrir sur ce compositeur? Que peut-on lire à son sujet? Quelles sont les sources disponibles au futur chercheur qui permettront d'analyser le discours sous-jacent à ses œuvres? Quelques travaux d'envergure ont été réalisés depuis quelques années. Nous pensons,

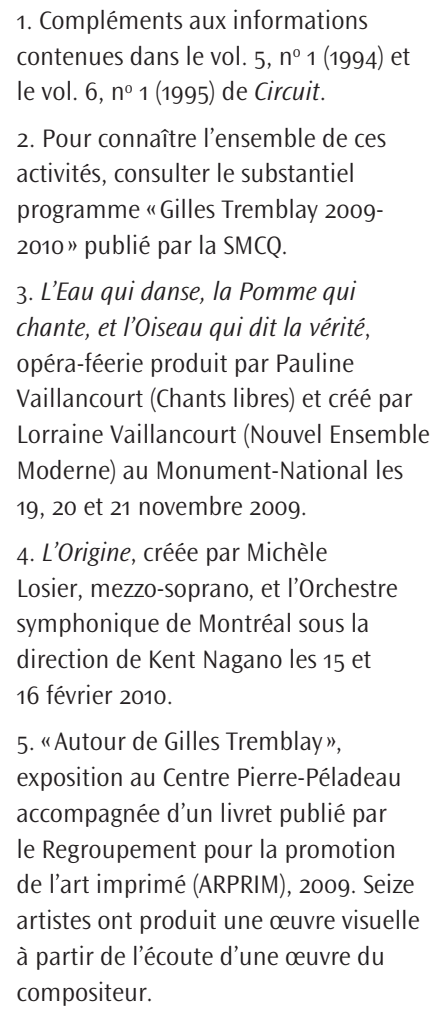


6. Le CMC du Québec a commandé au compositeur Serge Arcuri une adaptation pour harmonie d'extraits des Vêpres de la Vierge de Gilles Tremblay. La première a eu lieu le 20 novembre 2009 lors du Congrès annuel de la FAMEQ.

7. Au cours de cette journée, les participants ont entendu les propos de Walter Boudreau, Sylvain Caron, Sébastien Leblanc-Proulx, MarieThérèse Lefebvre, Isabelle Panneton, Vincent Ranallo, François Tousignant.

8. André Villeneuve (1983), "Souffles (Champs II) de Gilles Tremblay". Travail présenté au concours d'analyse, Conservatoire de musique de Montréal.

9. Vincent Ranallo (2006), "L'écoute créatrice dans l'orientation spirituelle de l'œuvre de Gilles Tremblay". Document de recherche soumis lors de l'examen de synthèse au programme de doctorat, Faculté de musique, Université de Montréal (archives de l'auteur). Un extrait de ce document est publié dans ce numéro de Circuit.

10. Daniel Turp (2009), "Gilles Tremblay et le devenir musical du Québec: un homme d'idées", de convictions et de projets. Travail de session, cours d'Histoire de la musique au Canada, automne (publié sur le site: danielturpqc.org/bloguelyrique/). entre autres, aux recherches d'André Villeneuve', de Vincent Ranallo9 et de Daniel Turp ${ }^{10}$. Cependant, la recherche sur l'esthétique et sur les œuvres de Gilles Tremblay et, d'une manière plus globale, les études visant à mettre en contexte son insertion dans l'histoire culturelle du Québec demeurent encore trop peu nombreuses, probablement parce que l'inventaire des sources documentaires est incomplet et que certaines sont plus ou moins accessibles. Nous pensons plus particulièrement ici aux documents conservés dans ses propres archives: notes de cours (comme étudiant, puis professeur), esquisses et autres documents préparatoires à la réalisation de ses œuvres, et surtout la correspondance qui, si on s'en tient aux lettres reçues, laisse entrevoir un travail immense pour répertorier celles qu'il a adressées à de nombreux amis et collègues ${ }^{11}$.

Pour ce qui est de la documentation accessible, nous avons publié dans des numéros précédents de la revue Circuit des listes de ses œuvres, écrits et entretiens radiophoniques (vol. $5, n^{\circ} 1$ ) ainsi qu'une bibliographie/discographie (vol. 6, n ${ }^{\circ}$ 1). Nous avions choisi de présenter ces documents dans un ordre chronologique de manière à faire ressortir l'évolution des propos de et sur Gilles Tremblay au fil de ses réflexions et de sa production. À l'invitation du rédacteur en chef de la revue, Jonathan Goldman, nous proposons dans ce numéro une mise à jour de ces informations, en respectant les mêmes catégories et le même ordre chronologique.

Outre les réflexions publiées par le compositeur, une grande partie de ces informations proviennent d'entretiens radiophoniques, plus difficiles à consulter. Afin d'en faciliter l'accessibilité, nous avons procédé à la transcription de la plupart des grandes entrevues qu'a données Tremblay au cours de sa carrière ${ }^{12}$. L'ensemble de ces documents qui couvrent la période de 1958 à 2010 (à l'exception de son étude sur Guillaume de Machaut, écrite en 1956) a été déposé au Centre de musique canadienne à Montréal.

La lecture chronologique des textes de Tremblay permet de mettre en évidence plusieurs thèmes dont l'importance varie en fonction de la période où ils apparaissent. Ainsi peut-on observer des liens entre sa pensée esthétique et les œuvres qui en découlent. Les fondements de sa spiritualité et de sa conception du sacré relié à la création musicale, sa quête de racines ou ses interrogations sur «l'origine» comme moteur essentiel de son inspiration, sa manière de s'insérer dans une histoire musicale continue, sans ruptures, ses réflexions sur la nature, le son et le silence comme modèles formels, sa conviction (utopique?) qu'une partie de la responsabilité du compositeur puisse être partagée avec l'interprète, ou enfin, ses prises de position engagées politiquement démontrant sa volonté de s'inscrire dans la réalité présente, 
actuelle, sont autant de questions que soulève la lecture de ces documents' ${ }^{13}$. Ce ne sont là, bien sûr, que quelques pistes qu'un futur chercheur aura le loisir d'approfondir, en attendant que puisse être rassemblée en un même lieu l'importante documentation archivistique du compositeur.

Parmi ces transcriptions d'entrevues radiophoniques que nous avons évoquées, nous proposons au lecteur, dans la rubrique «Document» de ce numéro, celle réalisée à Radio-Canada par Jean Lesage les 10, 11 et 12 février 1997 au moment où le compositeur annonçait sa retraite de l'enseignement. Elle résume à elle seule les grands axes de la pensée de Gilles Tremblay.

\section{b) Catalogue des œuvres ${ }^{14}$}

\section{D'une goutte... pour sgouglou}

Le «sgouglou», instrument microtonal inventé par Jean-François Laporte. Commande du groupe TUYO. Création par Carol Bergeron.

\section{L'Arbre de Borobudur}

Pour gamelan javanais degung (8 exécutants), cor, contrebasse, ondes Martenot, 2 harpes, 2 percussions. Commande de la Société de musique contemporaine du Québec grâce à une subvention du Conseil des Arts du Canada. Création à Montréal, en 1994, par The Evergreen Gamelan Club et l'Ensemble de la SMCQ sous la direction de Walter Boudreau.

\section{Traversée}

Concerto pour flûte et orchestre. Commande de Robert Aitkin avec l'aide du Conseil des Arts du Canada. Création le 3 novembre 1997 à Toronto, Robert Aitken, fûte et Esprit Orchestra sous la direction d'Alex Pauk.

\section{L'espace du cœur (Miron - Machaut)}

Pour 12 ou 24 voix mixtes et percussion. Commande de Pro Coro Canada avec l'aide du Conseil des Arts du Canada. Création le 15 septembre 1997 au Francis Winspear Centre for Music (version 24 voix): Pro Coro Canada, Trevor Brandenburg, percussion, sous la direction d'Agnès Grossman.

\section{Les pierres crieront}

Pour violoncelle solo principal et grand orchestre: violoncelle solo / 4 flûtes (incluant un piccolo), 4 hautbois (incluant un cor anglais), 5 clarinettes (incluant une clairennette en mi bémol et une clarinette basse), 4 bassons (incluant un contrebasson) / 4 trompettes (incluant une trompette en ré), 4 trombones (incluant un trombone basse) / timbales, 4 percussions / piano / cordes. Commande de Radio-France. Création le 19 mai 2001 par Jérôme
11. Nous avons établi une première liste de correspondants (dont le compositeur a reçu trois lettres ou plus). Parmi les Québécois, nous notons: Noël Audet, Claude Beauregard, Louise Bessette, Richard Boucher, Marc Chapdelaine, Gabriel Charpentier, Jean PapineauCouture, Yves Daoust, Serge Garant, Bruce Mather, François Morel, Fernand Ouellette, Isabelle Panneton, Wilfrid Pelletier, Clermont Pépin, Serge Provost Rober Racine, Hubert Reeves, R. Murray Schafer, Paul Vanier, s.j., Claude Vivier. Parmi d'autres personnalités, mentionnons: Gilbert Amy, Claude Baliff, André Boucourechliev, Pierre Boulez, Daniel Charles, Edmond Costère, Xavier Darasse, Jean-Claude Éloy, Claude Helffer, François Huang, Daniel Lesur, Yvonne et Jeanne Loriod, Maurice Martenot, Olivier Messiaen, Makoto Shinohara, Yuji Takahashi, Edgard et Louise Varèse.

12. À l'exception des entrevues qui ont eu lieu lors de la diffusion d'une œuvre où le compositeur reprend essentiellement ses notes de programme déjà publiées dans les partitions. Nous remercions Sébastien Leblanc-Proulx pour son aide précieuse à la réalisation de ces transcriptions.

13. Nous publierons dans la prochaine parution des Cahiers des Dix, n 64 (2010) une analyse sur les fondements de sa pensée religieuse.

14. Complément à Circuit, vol. 5, nº 1 (1994), p. 69-72 (www.erudit.org/revue/ circuit/1994/v5/n1/). 
Pernoo, violoncelle, et l'Orchestre national de France sous la direction de Luca Pfaff.

1999. À quelle heure commence le temps...

Monodrame lyrique sur un poème de Bernard Lévy. Pour baryton-basse, piano principal, et quinze instrumentistes. Commande du Nouvel Ensemble Moderne avec l'appui du Conseil des Arts du Canada. Création le 27 novembre 1999 par Michel Ducharme, baryton-basse solo, et le Nouvel Ensemble Moderne sous la direction de Lorraine Vaillancourt.

2000. La Symphonie du Millénaire (partie essentiellement consacrée à la Manécanterie des Petits chanteurs du Mont-Royal), œuvre collective de 19 compositeurs

Commande de la SMCQ. Création à Montréal, en juin 2000, sous la direction de Walter Boudreau.

2000. L'appel de Kondiaronk: symphonie portuaire

Pour sirènes de bateaux et deux locomotives. Commande de la Société RadioCanada.

2001. Croissant

Pour quatuor à cordes. Commande du New Music Concerts avec l'aide du Conseil des Arts du Canada. Création le 19 novembre, Massey Hall NUMUFEST, Toronto, par le Penderecki String Quartet.

\section{En partage}

Concerto pour alto et orchestre. Alto soliste / 3 flutes (incluant un piccolo), 3 hautbois (incluant un cor anglais), 3 clarinettes (incluant une clarinette basse), 3 bassons (incluant un contrebasson) / 4 cors, 3 trompettes, 3 trombones, 1 tuba / 3 percussions / cordes. Commande de l'OSM et de Charles Dutoit. Création les 8 et 9 octobre 2002 par Rivka Golani, alto, et l'OSM. Direction: Asher Fisch.

\section{L'infiniment petit}

Pour piano en seizième de ton. Commande de Bruce Mather avec l'aide du Conseil des Arts du Canada. Création : le 29 janvier 2004 à la Chapelle historique du Bon-Pasteur. Bruce Mather, piano.

\section{Chants convergents}

Pour voix de soprano, clarinette en si bémol, deux percussions et piano. Commande de la Faculté de musique de l'Université de Toronto. Création: 17 mars 2004, Walter Hall, Université de Toronto, par Kristin Mueller, 
soprano; Rob Spady, clarinette; Trent Petrussian, percussion; Tim Francom, percussion; Brian Harman, piano. Direction Gary Kulesha.

\section{Acht Flöten Fest für Robert Aitken in Freiburg}

Pour 8 grandes flûtes. Création: été 2004 à Freibourg.

\section{4-2007. L'Eau qui danse, la Pomme qui chante, et l'Oiseau qui} dit la vérité.

Opéra-féerie sur un livret de Pierre Morency (d'après Madame d'Aulnoy). Récitant, baryton / basse / soprano léger / contralto / alto / mezzo-soprano / ténor / deux barytons / haute-contre / danseuse / acrobate. Orchestre de 25 instrumentistes: flûte, petite flûte, hautbois, clarinette en si bémol, clarinette basse, basson / cor, trompette, trombone basse / 3 percussions / piano / cordes (8 violons, violoncelle, 3 contrebasses). Commande de Chants Libres avec l'aide du Conseil des Arts du Canada et du Conseil des arts et des lettres du Québec. Création en novembre 2009 .

\section{Levées}

Pour quatuor de saxophone. Commande du quatuor de saxophones QUASAR avec l'aide du Conseil des Arts du Canada. Création: le 19 février 2009, salle Pierre-Mercure, dans le cadre du Festival international Montréal / Nouvelles musiques (MNM).

\section{Musiques de l'eau}

Pour piano. Commande du Conservatoire de musique du Québec.

\section{L'Origine}

Pour baryton et orchestre. Commande de L'OSM. Création sous la direction de Kent Nagano le 15 février 2010.

\section{c) Chronologie des écrits (articles et entretiens radiophoniques) ${ }^{15}$}

1974. Jean Deschamps interroge Gilles Tremblay, suivi de la diffusion de Cantique de durées, CBC-TV, 13 janvier (une copie de l'enregistrement a été déposée au Centre de musique canadienne à Montréal).

1979. Documentaire Gilles Tremblay. Animatrice: Norma Beecroft. Émission Two New Hours, CBC Broadcast, 6 mai (une copie de l'enregistrement a été déposée au Centre de musique canadienne à Montréal).

1982. Entrevue de Louise Bail avec Gilles Tremblay, émission Musique de Canadiens (Radio-Canada), réalisateur Normand Plante, Radio-Canada, le 16 mai (une copie de cette série a été déposée au Centre de musique canadienne à Montréal).
15. Complément à Circuit, vol. 5, n $\mathrm{n}^{\circ} 1$ (1994), p. 73-76 (www.erudit.org/revue/ circuit/1994/v5/n1/). 
16. Actuellement dans la famille, les archives du compositeur seront éventuellement déposées dans une institution publique.
1983. Entretien de Maryvonne Kendergi avec Gilles Tremblay, Anthologie de la musique canadienne, vol. 16, Radio-Canada International.

1986. Hommage à Serge Garant. Texte lu lors des funérailles de Serge Garant, à la cathédrale de Sherbrooke, le 8 novembre. Archives du compositeur ${ }^{16}$. 1989. "La voix, le bel canto et la création musicale». Communication présentée lors du Festival international de Montréal, table ronde réunissant Gilles Tremblay, Jacques Hétu, John Rea, et animée par Pierre Lapalme, le 11 septembre (archives du compositeur).

Homélie de la vigile de Pâques, le 30 mars. Archives du compositeur.

1991. Texte de la pochette du disque de la pianiste Louise Bessette consacré aux œuvres de Charles Ives et Olivier Messiaen, juin. Archives du compositeur.

1992. Émission animée par Jean Lesage, avec des compositeurs et musicologues qui répondent à la question: "Que représente Olivier Messiaen pour vous? », émission Musique actuelle (Radio-Canada), spécial Olivier Messiaen, le 3 mai.

1992. Religion, musique et temps à travers l'expérience de la composition des Vêpres de la Vierge. Communication présentée à la Faculté de théologie de l'Université de Montréal, à l'occasion du colloque «Société, culture et religion dans le Montréal métropolitain », le 20 mai.

1992. Portrait du compositeur et professeur Gilles Tremblay. Émission

Musique actuelle (Radio-Canada) réalisée par Laurent Major lors du concert Euro-Radio, le 12 octobre à l'église St-Jean-Baptiste de Montréal. Témoignages d'Hubert Reeves, François Morel, Claude Helffer, Claude Baliff, Xavier Darasse, Iannis Xénakis, Gilbert Amy, Bruce Mather, Jeanne Renaud, Pauline Vaillancourt et Maryvonne Kendergi.

1992. Entrevue de Marie-Claude Lavallée avec Gilles Tremblay sur son œuvre AVEC, Wampum symphonique. Émission Bonjour Musique (RadioCanada), le 12 octobre.

1992. Entrevue de Françoise Davoine avec Gilles Tremblay sur son œuvre AVEC, Wampum symphonique. Émission Radio-Concert (Radio-Canada), le 12 octobre.

1993. Entrevue de Françoise Davoine avec Gilles Tremblay sur Mauricio Kagel. Émission Radio-Concert (Radio-Canada), le 22 janvier.

1993. Texte sur la paix. Adresse aux Artistes pour la paix, le 5 mars. Archives du compositeur.

1993. Table ronde autour de l'œuvre de Iannis Xenakis, animée par Serge Provost et réunissant Robert Dupuis, Jean Letourneux, Michel Longtin et Gilles Tremblay. Émission Musique actuelle (Radio-Canada), le 18 avril. 
1993. Entrevue téléphonique de Serge Provost avec Gilles Tremblay sur son œuvre pour orgue, Vers les étoiles. Émission Musique actuelle (RadioCanada), le 2 mai.

1993. Table ronde animée par Mario Paquet sur la situation actuelle et l'avenir de la musique contemporaine, et sur le métier de compositeur, avec Serge Arcuri, Walter Boudreau, Linda Bouchard, Jose Evangelista, Jacques Hétu et Gilles Tremblay. Émission Musique actuelle (Radio-Canada), le 23 mai. 1993. Gilles Tremblay: un univers sonore (à propos de l'œuvre pour orgue Vers une étoile créée par Bernard Fouccroule), animée par Jean Lesage. Émission télévisuelle réalisée par James Dormeyer dans le cadre des Beaux Dimanches (Radio-Canada), le 31 octobre.

1993. Hommage à Jean-Marie Beaudet, transmis à Pierre Beaudet qui préparait à cette époque une biographie de son frère, le 15 novembre. Archives du compositeur.

1994. Portrait d'Olivier Messiaen. Témoignages de plusieurs intervenants recueillis par Catherine Perrin, dont celui de Gilles Tremblay. Émission télévisée française En avant la musique, le 10 avril.

1994. "Silences, silence», Actes du colloque sur l'écologie sonore tenu à l'Université Laval le 9 avril, sous la direction de Raymond Ringuette, Recherche en éducation musicale au Québec, volume 13, novembre 1994, 12-19.

1995. Hommage à Raynald Arseneault, lors des funérailles à l'église SaintMarc de Rosemont, le 15 janvier. Archives du compositeur.

1995. Hommage à Raynald Arseneault. Témoignage de Gilles Tremblay interrogé par Françoise Davoine. Émission Radio-Concert (Radio-Canada), le 30 janvier.

1995. Serge Provost s'entretient avec Gilles Tremblay sur son œuvre L'arbre de Borobudur. Émission Radio-Concert (Radio-Canada), le 13 mars.

1995. «Un vingt-quatrième de seconde », hommage à Maurice Blackburn, à la demande de Réal Larochelle, le 8 mai. Archives du compositeur.

1995. Le Conservatoire se raconte. Entrevue avec Serge Provost, Chapelle historique du Bon-Pasteur, le 24 mai. Archives du compositeur.

1996. Le compositeur explique le sens du titre de son œuvre Katadrone interprétée par l'Orchestre symphonique de Québec sous la direction de Bramwell Tovey. Émission Radio-Concert (Radio-Canada), le zo mars.

1996. Témoignage lors d'une fête soulignant le centenaire de naissance de Mgr Félix-Antoine Savard, le 31 août. Archives du compositeur.

1997. Qui êtes-vous? Gilles Tremblay, compositeur. Série de trois émissions animées par Jean Lesage, Radio-Canada, les 10, 11 et 12 février ${ }^{17}$.
17. [ndlr] Une transcription de cette série d'entretiens se trouve dans le présent numéro. Voir la rubrique "Document". 
1997. Georges Nicholson discute des Vêpres de la Vierge avec Gilles Tremblay et Walter Boudreau. Émission De bouche à oreille (Radio-Canada), le 7 décembre.

1997. Hommage à alcides lanza lors de la réception du prix qui lui a été conféré par l'OEA et le Conseil inter-américain de la musique, le g décembre. Archives du compositeur.

1997. Présentation de Maryvonne Kendergi au prix du Gouverneur général du Canada. Archives du compositeur.

1998. Allocution de Gilles Tremblay lors de la remise du prix Serge-Garant, le 19 février. Archives du compositeur.

1999. Après la mort, émission animée par Liza Frulla avec la participation de Marie-Chantal Beaunoyer, Benoît Lacroix, Jacques Sénécal et Gilles Tremblay. Émission télévisée (Radio-Canada), le $1^{\text {er }}$ avril.

1999. Interrogé par Winston McQuade, Gilles Tremblay évoque son approche du texte À quelle heure commence le temps? sur lequel il a composé la musique. Il décrit le résultat final. Bernard Lévy parle de sa rencontre sur le plan artistique avec le compositeur. Il décrit le contexte qui a favorisé leur collaboration et les adaptations qui ont dû être faites au texte afin qu'il soit en harmonie avec la musique. Émission Multimédiart, Radio-Canada, le 26 novembre 2000. À l'occasion de la 6e édition des Symphonies portuaires, Gilles Tremblay raconte à Joël Le Bigot l'histoire de sa symphonie L'Appel de Kondiaronk. Émission Samedi et rien d'autre, Radio-Canada, le 26 février.

2000. En direct de Pointe-à-Callière, Gilles Tremblay présente sa symphonie portuaire L'Appel de Kondiaronk à l'émission Un dimanche à la radio, Radio-Canada, réalisée par Hélène Prévost, le 27 février.

2000. Gilles Tremblay, «La musique et le sacré», Le Devoir, le 22 avril.

2000. Émission spéciale consacrée au $4^{\mathrm{e}}$ Gala des prix Opus. Extraits d'une rencontre avec Gilles Tremblay qui commente son œuvre À quelle heure commence le temps? Émission Concert et compagnie, le 20 novembre.

2002. Gilles Tremblay raconte la genèse de sa composition Les Vêpres de la Vierge et explique comment il a opéré une synthèse entre le chant grégorien et l'instrumentation moderne dans cette composition. Série radiophonique Musiques d'un siècle. Émission consacrée à l'inspiration religieuse, produite par Luc Terrapon, Espace 2, Radio Suisse Romande, le 7 janvier. Émission produite à partir de documents d'archives. Propos de Nicolas Nabokov, Igor Stravinsky, Francis Poulenc, Frank Martin, Gilles Tremblay et Olivier Messiaen. 
2002. Gilles Tremblay, «Pour un débat ouvert sur la culture à la radio », lettre adressée au Devoir, le 19 juillet. Pour connaître l'ensemble des interventions lors de ce débat lancé par Jean Larose au journal Le Devoir, le 22 juin, consulter le site suivant: archives.vigile.net/ds-medias/docs/oz-6-22-larosemedias.html.

2002. Gilles Tremblay, «Hommage à Iannis Xenakis à l'occasion du premier anniversaire de sa mort», Études, 2002, vol. 2, tome 396, p. 259-262.

2002. Jean-Philippe Trottier s'entretient avec Gilles Tremblay, L'Agora, automne.

2002. Georges Nicholson interroge Gilles Tremblay à l'occasion de son $70^{\mathrm{e}}$ anniversaire. Émission radiophonique (Radio-Canada), les 6 et 13 octobre. 2002. Préface au livre de Louise Bail, Maryvonne Kendergi. La musique en partage, Montréal, Éditions HMH.

2002. À l'occasion de la remise du prix Hommage au Gala des prix Opus du Conseil québécois de la musique, Gilles Tremblay fait un bilan de sa carrière. Émission Concerts sans mesure, Radio-Canada, le 25 novembre.

2002. Hommage à Olivier Messiaen, à l'occasion du $10^{\mathrm{e}}$ anniversaire de son décès. Interventions et documents d'archives de Jean Boivin, Françoise Aubut, Clermont Pépin, Jacques Hétu et Gilles Tremblay. Émission RadioConcerts, Radio-Canada, le 2 décembre.

2003. Portraits: Gilles Tremblay, le compositeur s'entretient avec Jean-Paul Bataille. Émission radiophonique, Radio-Canada, le 6 juillet.

2003. Eitan Cornfield interroge Gilles Tremblay. Portraits de compositeurs canadiens, Centredisques CMCCD-9003. Texte de la traduction française (2005) accessible au Centre de musique canadienne, www.musiccentre.ca ou www.centremusique.ca.

2003. Présentation du pianiste Miguel Angel Estrella, concert-bénéfice du Mouvement international ATD Quart Monde, le 13 avril. Archives du compositeur.

2004. Hommage à Roger Matton, à la demande de Victor Bouchard, le 25 juin. Archives du compositeur.

2004. Hommage à Claude Helffer, Le Devoir, le 7 novembre.

2005. Allocution lors de l'annonce du concours international de composition présidé par l'Orchestre symphonique de Montréal, le 29 mars. Archives du compositeur.

2005. Hommage à Pierre Mollet, le 3 avril. Archives du compositeur.

2006. Radio-documentaire «La mort du silence», réalisé par Mario Proulx. Parmi les intervenants, Gilles Tremblay. Il discute des conséquences de 
18. Complément à Circuit, vol. $6, \mathrm{n}^{0} 1$ (1995), p. 57-60 (www.erudit.org/revue/ circuit/1995/v6/n1/index.html). l'omniprésence du bruit dans nos sociétés. Émission Des idées plein la tête - un autre regard, Radio-Canada, le 9 janvier.

2006. Composer?! Portraits de compositeurs: Gilles Tremblay. DVD produit par la Société de musique contemporaine du Québec en collaboration avec ÈS ART et Productions Éneri, mars.

2006. À titre de président d'honneur du Prix d'Europe 2006, Gilles Tremblay répond aux questions de Joël Le Bigot à l'émission Samedi et rien d'autre, Radio-Canada, le $z$ juin.

2007. Gilles Tremblay, «Hommage à François Morel». Programme du concert-hommage à François Morel par le Chœur de Métal, salle Pollack, Montréal, le 15 juin.

2007. Gilles Tremblay, «Appauvrissement culturel», lettre adressée au journal Le Devoir, le 11 juillet (après le départ de l'animateur Georges Nicholson à l'Opéra du samedi).

2007. Portrait du compositeur Gilles Tremblay, récipiendaire du Prix Cino del Duca décerné par l'Académie des beaux-arts (France). Émission Soirées classiques animée par Mario Paquet, Radio-Canada, le 14 novembre.

2007. Texte soulignant les vingt ans de l'Ensemble contemporain de Montréal. Archives du compositeur.

2008. Gilles Tremblay, «Hommage à Stockhausen », Les Cahiers de la Société québécoise de recherche en musique, vol. 10, nº 1, p. 89-9o.

2008. Hommage de Gilles Tremblay à Olivier Messiaen. www.automnemessiaen.net.

2008. Gilles Tremblay, «Envoi » : conférence d'ouverture au colloque Les écrits des compositeurs depuis 1850, organisé par Michel Duchesneau et Jean-Jacques Nattiez, Faculté de musique, Université de Montréal. 12-15 mars. Vidéo déposé au Centre de documentation de l'OICRM.

2008. Gilles Tremblay explique à Luc Beauséjour le titre de son œuvre Cèdres en voiles lors d'un concert au Domaine Forget. Émission La radio des festivals, Radio-Canada, les 4 et 13 août.

2009. «Le rôle de l'artiste dans la société », entrevue de Réjean Beaucage avec Gilles Tremblay, Cahier MNM, p. 11.

\section{d) Bibliographie chronologique ${ }^{18}$}

Martin, Paul (1962), «À la tête de proue de la jeune musique canadienne: Gilles Tremblay», Le Devoir, le 4 juillet.

Vallerand, Claudine (1962), «Nos "Mozart assassinés" », L’opinion de nos lecteurs, Le Devoir, le 31 juillet. 
Chamberland, Paul (1962), «Réponse à Mme Claudine Vallerand », Courrier des lecteurs, Le Devoir, le 15 août.

Vivier, Claude (1991), «À propos de Gilles Tremblay», Circuit, vol. 2, nº 1-2.

Dubois, Anne, réalisatrice (1992), «Hommage à Gilles Tremblay», Musique en fête, Radio-Canada, le 7 septembre.

Gingras, Claude (1993), «Foccroulle! L'orgue contemporain », La Presse, le 6 mai.

Bergeron, Carol (1993), «L'orgue bien vivant», Le Devoir, le 7 mai.

Olivier, Dominique (1995), «Orient-Express», Voir, mars.

Kaptainis, Arthur (1995), "Rarely the Twain did Meet: East meets West as SMCQ teams up with Toronto's Evergreen Club», The Gazette, le 10 mars.

Ouellette, Fernand (1996), «L'oreille intérieure de Gilles Tremblay », in En forme de trajet: essais, Éditions Le Noroît, p. 75-77.

Boivin, Jean (1996), "Messiaen et le Québec», Revue de musique des universités canadiennes, 17/1, p. 72-97.

Leroux, Robert (1997), «Éloge de Gilles Tremblay», texte du président du jury lors de la remise du prix Serge-Garant. Archives de l'auteur.

Trudel, Clément (1999), "Gilles Tremblay: compositeur pour l'éternité. L'artiste souhaite un débat sur la culture », Le Devoir, le 8 mars.

Trudel, Clément (1999), «Le chantier du reclus», Le Devoir, le 13 septembre.

Duchesneau, Michel (1999), "Gilles Tremblay: musique pour la vie », BOULiANe, Denys (dir.), Présence de la musique québécoise: vingt-deux portraits instantanés, Programme du Festival Présences 99 de Radio-France en collaboration avec la SMCQ et le NEM, Montréal, p. 83-86.

Gingras, Claude (2002), «OSM: Une création de Tremblay ce soir», La Presse, le 8 octobre.

Gingras, Claude (2002), "OSM: Watts sauve le concert», La Presse, le 9 octobre.

Tousignant, François (2002), "Une création tiède et un bouche-trou », Le Devoir, le 9 octobre.

BAIL, Louise (dir.) (2003), «Hommage à Gilles Tremblay», Les Cahiers de la Société québécoise de recherche en musique, vol. 7, nº 1-2.

Ranallo, Vincent (2006), L'écoute créatrice dans l'orientation spirituelle de l'œuvre de Gilles Tremblay. Document de recherche soumis lors de l'examen de synthèse au programme de doctorat, Faculté de musique, Université de Montréal (archives de l'auteur).

Ranallo, Vincent (2007), "L'écoute créative dans les mobiles de Gilles Tremblay », La Scena Musicale, avril, p. 18-19. 
Huss, Christophe (2007), «Esprit frappeur», Le Devoir, le 7 décembre.

Gingras, Claude (2008), «OSM: Deux symphonies et deux nouveautés», La Presse, le 26 février.

Gingras, Claude (2008), « Molinari-Bessette autour de Messiaen », La Presse, le 29 mars.

RichaRD, Robert (2009), «Éblouissement. L'œuvre du compositeur Gilles Tremblay », Liberté, vol. 51, n 1 .

Boudreau, Walter et Gingras, Pierrette (dir.) (2009), programme des événements entourant la Série Hommage à Gilles Tremblay, Montréal, Société de musique contemporaine du Québec (SMCQ).

Renaud, Lucie (2009), "Le passeur: Gilles Tremblay», La Scena Musicale, octobre, vol. 8, nº 11 .

Collectif (2009), Autour de Gilles Tremblay, ARPRIM (Regroupement pour la promotion de l'art imprimé), catalogue d'exposition, Chapelle historique du Bon-Pasteur.

Ouimet-Lamothe, Sophie (2009), «Un conte féerique contemporain », La Presse, le 14 novembre.

Huss, Christophe (2009), «L'opéra-féerie de Gilles Tremblay», Le Devoir, le 14 novembre.

Huss, Christophe (2009), «Musique classique: féerie visuelle », Le Devoir, le 20 novembre.

Gingras, Claude (2009) «Un opéra pour l'œil », La Presse, le 21 novembre.

Kaptainis, Arthur (2009), «L'Eau qui danse, la Pomme qui chante et l'Oiseau qui dit la vérité», The Gazette, le 20 novembre.

Turp, Daniel (2009), Gilles Tremblay et le devenir musical du Québec: un homme d'idées, de convictions et de projets. Travail de session, cours d'Histoire de la musique au Canada, automne (archives de l'auteur sur le site: danielturpqc.org/bloguelyrique/ ).

DéCARy, Marie (2009), Au pays de l'émerveilleux Gilles Tremblay, livre jeunesse illustré par Élisabeth Eudes-Pascal, production de la SMCQ, 2009.

Huss, Christophe (2010), «Le piano lumière », Le Devoir, le 16 février (comprend aussi un commentaire sur la création de L'Origine). Sur le site du journal, on peut également lire les réactions de Michel Gonneville, Simon Bertrand et Mario Gauthier à cette critique.

Huss, Christophe (2010), «Lumières dans la nuit» [à propos des Vêpres de la Vierge], Le Devoir, le 17 avril, B-9.

Gingras, Claude (2010), «SMCQ: finies, les Vêpres», La Presse, le 16 avril. 


\section{e) Discographie ${ }^{19}$}

1994. Envol, Centredisques CMCCD 5094. Aubes (1990), Envol (1986), «... le sifflement des vents porteurs de l'amour...» (1971). Ensemble Pierrot, dir. David Currie.

1998. Les Vêpres de la Vierge (1986), Analekta FL 2 3102. Société de musique contemporaine du Québec, Chœur de chambre de l'OSM, dir. Walter Boudreau.

2003. Ovation, Volume 3, CBC Records PSCD 2028-5. Fleuves (1976), L'arbre de Borobudur (1994), Les pierres crieront (1998). Ensembles divers.

2003. Canadian Composers Portraits: Gilles Tremblay, Centredisques CMCCD 9003. Phases (1956), Réseaux (1958), Jeux de solstices (1971), Oralléluiants (1975), L'espace du cour (1997), Croissant (2001), ensembles divers.

2006. À quelle heure commence le temps? (1999), Atma ACD 22376. Nouvel Ensemble Moderne, dir. Lorraine Vaillancourt.

2006. Composer?! 12 portraits. DVD réalisé par Irène Messier et Anne-Marie Messier. SMQC en coproduction avec ÈS ARTS et Productions Énéri.

2007. Le Signe du lion, Centredisques CMCCD 12507. Le Signe du lion (1981), Solstices (1971), Envoi (1982), Ensemble Aventa, dir. Bill Linwood.
19. Complément à Circuit vol. 5, $\mathrm{n}^{0} 1$ (1994), p. 77-78 (www.erudit.org/revue/ circuit/1994/v5/n1/index.html). 